\title{
8 Cross-Categorical Value Comparisons
}

\author{
Krister Bykvist
}

\section{Introduction}

Without blinking an eye, we make comparisons across distinct value categories. Here are some examples. ${ }^{1}$

(1) This table is a better table than Trump is a president. $^{2}$

(2) You are more prudent than you are moral.

(3) I am more kind than I am beautiful.

This is puzzling since "better table," "more prudent," and "more kind" cannot be read in the usual ways here: "being better as a table," "showing a higher degree of prudence," and "showing a higher degree of kindness," respectively. This has prompted some linguists to think that these constructions are anomalous in some sense, ungrammatical or nonsensical. Others have claimed they are grammatical and meaningful but only metaphorical or metalinguistic (e.g., it is more appropriate to say "this is a good table" than to say "Trump is a good president"). Some linguists, such as Alan Bale (2006, 2008) and Ewan Klein (1980), however, have offered what they think is a meaningful non-metaphorical interpretation. Except for a few brief comments, philosophers have so far been silent on this perplexing issue, which is especially odd since philosophers thrive on puzzles. ${ }^{3}$

In this chapter, I shall argue against the idea that the meaningfulness of cross-categorical comparisons (sometimes called "indirect" or "interadjectival" comparisons) is an illusion. I shall also argue against the existent linguistic ideas about how to make these comparisons meaningful. My focus throughout will be on cross-categorical value comparisons. I shall give the rough contours of a new theory of such comparisons (with a hint on how to extend them to their non-evaluative counterparts) and show that they are normatively relevant. In particular, I shall suggest how they can be usefully invoked when comparing well-being across people, when assessing the fittingness of attitudes, and when making cross-theory value comparisons in the context of acting under evaluative uncertainty

DOI: $10.4324 / 9781003148012-12$ 
("What should I do when I am not certain about the values of my alternatives?”). If I am right, then there is a need to posit a new nonstandard value relation or, more exactly, a family of cross-categorical betterness and equality relations.

Before we start, I need to make an important clarification. I am interested in philosophical analyses of the concept of cross-categorical comparisons: a priori, necessarily true biconditionals in which the left-hand claim, if true, holds in virtue of the right-hand claim. This is not the typical focus in linguistic analysis, which only aims to provide the truth conditions of a natural language sentence. To draw on linguistic proposals, I will therefore read them as philosophical analyses. This also means that I will gloss over issues concerning the surface grammar of English, which often plays an important role in linguistics but less so in philosophical analysis.

\section{Cross-Categorical Comparisons in General}

What are cross-categorical comparisons (let us call them cross-cats for short, which also will remind us of their cross-breed nature)?

First, we can say that they have any of the following forms, where $\mathrm{F}$ and $\mathrm{G}$ are different gradable adjectives and $\mathrm{A}$ and $\mathrm{B}$ are nouns:

$x$ is $\mathrm{F}$-er/more $\mathrm{F}$ than $y$ is $\mathrm{G}$.

$x$ is $\mathrm{F}$-er/more $\mathrm{F}$ for an $\mathrm{A}$ than $y$ is $\mathrm{G}$ for a $\mathrm{B}$.

$x$ is an F-er A than $y$ is a $\mathrm{G} \mathrm{B}$.

However, not all constructions of these forms are cross-categorical in the sense that I am interested in. Take, for instance, this cross-adjectival sentence:

(4) This chair is taller than it is wide.

This is not cross-categorical in the relevant sense, since the tallness and the width of the chair can be directly compared in terms of length, in $\mathrm{cm}$, for example.

What I am after are constructions in which $\mathrm{F}$ and $\mathrm{G}$ are distinct adjectives that do not pick out directly comparable properties. Here are some non-evaluative examples:

(5) Einstein is more intelligent than I am beautiful.

(6) I am more angry than she is happy.

(7) He is taller for a Swede than he is heavy for a Swede.

For none of the pairs (intelligent, beautiful), (angry, happy), and (tall, heavy) are the elements directly compared on one common dimension, 


\section{Krister Bykvist}

and the same seems to hold for the evaluative examples listed at the beginning of the chapter: (good as a table, good as a president), (prudent, moral), and (kind, beautiful).

One could perhaps object here that appearances are misleading when it comes to the evaluative examples. It may seem as though the evaluative properties cannot be directly compared, but if we look closer, we can see that they can be so compared. For example, why not say that

(2) you are more prudent than you are moral, should roughly be understood as

(8) the degree to which I am prudent counts more toward my flourishing or overall virtuousness than the degree to which I am moral?

The idea is that being kind and being moral fall under the same covering value, counting toward my flourishing or overall virtuousness. ${ }^{4}$ This does not seem to be plausible, however. It is perfectly fine to say that

(9) you are more prudent than you are moral, but I have no idea about what makes your life flourish more.

Furthermore, there is nothing strange about saying that

$\left(9^{*}\right)$ it is unfortunate that you are more prudent than you are moral, since the degree to which you are moral counts more toward your flourishing than does the degree to which you are prudent.

Returning to the matter of delineating cross-categorical comparisons, we can see that, as Bale notes, one crucial difference between cross-adjectival statements that are and those that are not reducible to direct comparisons concerns their conceptual entailments.

(10a) I am taller than I am wide.

(10b) If I am very wide, then I am at least very tall.

(11a) Einstein is more intelligent than you are beautiful.

(11b) If you are very beautiful, then Einstein is at least very intelligent.

(12b) He is taller for a Swede than he is heavy for a Swede.

(12c) If he is very heavy for a Swede, he is at least very tall for a Swede.

Statement 10a does not entail 10b, for $10 \mathrm{a}$ can be true even if I am very wide for a man - say, $1 \mathrm{~m} 30 \mathrm{~cm}$ - and short for a man - say, $1 \mathrm{~m} 32$ $\mathrm{cm}$. All it takes for 10a to be true is that my height exceeds my width. In contrast, $11 \mathrm{a}$ entails $11 \mathrm{~b}$, and $12 \mathrm{a}$ entails $12 \mathrm{~b}$. That is why these informal inferences all seem fine. 
Einstein is more intelligent than you are beautiful.

You are very beautiful.

So

Einstein is at least very intelligent.

He is taller for a Swede than he is heavy for a Swede.

He is very heavy for a Swede.

So

He is at least very tall for a Swede.

The same kind of entailment holds for our evaluative examples:

(13a) This table is a better table than Trump is a president.

(13b) If Trump is a very good president, then this table is at least a very good table. (Given Trump's actual behavior, it is more appropriate to use a counterfactual conditional here!)

(14a) I am more selfish than you are moral.

(14b) If you are very moral, then I am at least very selfish.

(15a) You are more kind than you are brave.

(15b) If you are very brave, then you are at least very kind.

\section{Skepticism about Cross-Categorical Comparisons}

There is skepticism about whether we should take cross-cats literally. They have been accused of being ungrammatical, meaningless, metaphorical, and metalinguistic. So before we go on to discuss literal interpretations of cross-cats, we need to respond to these skeptical challenges. I think we can put the accusations of ungrammaticality and meaninglessness to one side since it seems obvious that we can use cross-cats grammatically and meaningfully. Furthermore, as we have seen, they also have clear and distinctive entailments.

According to the metaphorical interpretation, cross-cats only have metaphorical meaning. It is true that we sometimes use cross-cats to convey hyperbole:

(16) I am older than the universe is big.

(17) He is taller than the Great Pyramid of Khufu is heavy.

But it is one thing to say cross-cats can be used metaphorically and another to say that they can only be used in this way. For example, we do not seem to be forced to use the previously listed sentences metaphorically.

(1) This table is a better table than Trump is a president.

(2) You are more prudent than you are moral.

(3) I am more kind than I am beautiful. 


\section{Krister Bykvist}

They seem to have a literal meaning as well. I hope that the account of cross-cats I will present will validate this point - at least for evaluative cross-cats.

According to the metalinguistic idea, a cross-categorical sentence of the form

$x$ is $\mathrm{F}$-er/more $\mathrm{F}$ than $y$ is $\mathrm{G}$

should be understood as something like

it is more appropriate to say " $x$ is F" than to say " $y$ is G."

(The other constructions can be given analogous readings.) This account does not say much until one says why it is more appropriate to do this. One possibility is that it is more appropriate to say it because

$x$ is a clearer example of an $\mathrm{F}$ than $y$ is an example of a $\mathrm{G}$.

This approach does not work for all cross-cats, however, since it can be true that $x$ is F-er than $y$ is $\mathrm{G}$, even though $x$ is clearly not an F and $y$ is clearly not a $\mathrm{G}$

(18) I am taller than you are heavy

can be true even though I am a very short person, and thus clearly not a tall person, and you are a very light person, and thus clearly not a heavy person. Perhaps you are very light, and I am short but not very short.

Of course, much more can be said about this and other alternative metalinguistic approaches, but I hope that the account of cross-cats I will present shows that there is no need to "go metalinguistic" (at least not for evaluative cross-cats).

\section{Positional Accounts}

According to what I shall call positional accounts,

$x$ is F-er than $y$ is G iff $x$ has a higher position in the F-ranking than $y$ has in the G-ranking.

$x$ is equally as $\mathrm{F}$ as $y$ is $\mathrm{G}$ iff $x$ has the same position in the F-ranking as $y$ has in the G-ranking.

(The other constructions are given analogous readings.) Applied to our evaluative examples, this means that

This table is a better table than Trump is a president iff this table has a higher position in the good as a table ranking than Trump has in the good as a president ranking. 
You are more prudent than you are moral iff you have a higher position in the prudence ranking than you have in the morality ranking.

I am more kind than I am beautiful iff I have a higher position in the kindness ranking than I have in the beauty ranking.

This account has a ring of truth, but the crucial question is how to understand the talk about "higher position." Alan Bale (2006, 2008) spells it out as follows. Suppose we are considering $n$ items ranked in terms of F-ness and also $n$ items ranked in terms of G-ness. For each ranking, assign an item the ratio $n / n=1$ if it is on top in the ranking, the ratio $n-1 / n$ if it is second, $n-2 / n$ if it is third, and so on. In Bale's universal scale account,

$x$ is F-er than $y$ is $\mathrm{G}$ iff the ratio assigned to $x$ is greater than the ratio assigned to $y$.

$x$ is equally as $\mathrm{F}$ as $y$ is $\mathrm{G}$ iff the ratio assigned to $x$ is the same as the ratio assigned to $y$.

As it stands, this account is severely restricted, however.

First, it only works if both the F-ranking and the G-ranking are complete. But we seem to be able to make cross-categorical comparisons when at least one of the rankings has gaps. For example, we can say that:

(19) I am a better teacher than Talentlesso is an artist,

even if we assume that the good as an artist-ranking is incomplete. Perhaps Mozart cannot be said to be a better, worse, or equally as good artist as Michelangelo, to use a famous example. ${ }^{5}$

Second, it only works if the F-ranking and the G-ranking have the same number of compared items. To see this, suppose there are three Fs and five Gs. Then the second-best item in the F-ranking will be assigned twothird and the second-best in the G-ranking will be assigned two-fifths.

We do not want to say that the second-best item is F-er than another is $G$ just because the F-ranked items are less numerous. For example, we do not want to say that I am kinder than you are brave just because, even though we are both second-best in our respective rankings, there are more brave people than kind ones.

Third, it only works if there is a finite number of compared items. But we seem to be able to make true cross-cats when at least one of the rankings has an infinite number of items, for example, when one of the compared properties is unbounded:

(20) I am more moral than you (who are suffering immensely) are well off,

where being well off is an unbounded property (there is no limit to how well off you can be). 


\section{Krister Bykvist}

Perhaps these flaws can be fixed in a more sophisticated positional account. But there remains one problem that no positional account seems to be able to deal with. To see this, note first that any positional account must say that

if $x$ is on top in the F-ranking and $y$ is at the bottom in the G-ranking, then $x$ is higher up in the F-ranking than $y$ is in the G-ranking. ${ }^{6}$

Assume the top-ranked $x$ is very bad in the relevant F-sense and the bottom-ranked $y$ is very good in the relevant G-sense. Then it does not seem to be true to say that $x$ is F-er than $y$ is G despite the fact that $x$ is higher up in the F-ranking than $y$ is in the G-ranking. Suppose, for example, $a, b$, and $c$ are compared in terms of both being good as a philosopher and being kind. They are all very bad as philosophers, but $a$ is less bad than $b$, and $b$ is less bad than $c$. They are all very kind but $a$ is kinder than $b$ and $b$ is kinder than $c$. Then $a$ has a higher position in the good as a philosopher ranking than chas in the kindness ranking since $a$ is top ranked and $c$ is bottom ranked. But we do not want to say that $a$ is a better philosopher than $c$ is kind.

We can make a similar point without focusing on cases in which the polarity is different (very bad versus very good). Suppose $a, b, c$ are all kind but not very kind, and $a$ is kinder than $b, b$ is kinder than $c$. Suppose also that $a, b, c$ are all very beautiful, and $a$ is more beautiful than $b$, and $b$ is more beautiful than $c$. Then $a$ has a higher position in the kindness ranking than $c$ has in the beauty ranking, but we do not want to say that $a$ is kinder than $c$ is beautiful.

In fact, this example shows that Bale's account is in danger of being internally inconsistent. Remember that he wants to validate this inference:

$a$ is kinder than $c$ is beautiful.

$c$ is very beautiful.

\section{So}

$a$ is at least very kind.

If Bale wants to insist that $a$ is kinder than $c$ is beautiful because $a$ is higher up in the kindness ranking than $c$ is in the beauty ranking, then he can no longer accept the entailment he claimed was a characteristic feature of cross-cats.

To these objections, Bale could reply that he had in mind cases in which we consider and rank all brave people and all kind people. If we consider the maximal comparison class of all kind people, the topranked ones must be very kind and the bottom ranked not very kind. Similarly, if we consider the comparison class of all beautiful people, 
the top-ranked ones must be very beautiful and the bottom ranked not very beautiful. So it cannot be true that the top ranked in terms of kindness is not very kind and the bottom ranked in terms of beautiful is very beautiful.

But then we are back to the problem of unboundedness. This move does not work for cases in which there are no maximal or minimal elements, so the choice is between internal inconsistency or a severely restricted account. I hope we can do better and avoid this dilemma.

\section{Further Constraints on Evaluative Cross-Cats}

The previous examples show that we have further constraints on a viable account of evaluative cross-cats. Let us use "X-good," "X-bad," "X-neutral," "X-better" as short for "good in the relevant X-sense" (examples of instances would be "prudentially good" and "good as a table"), "bad in the relevant X-sense" (e.g., "prudentially bad" and "bad as a table"), "neutral in the relevant X-sense" (e.g., "finally neutral"), and "better in the relevant X-sense" (e.g., "prudentially better" and "better as a table"), respectively. We can now state the further constraints succinctly (where $F$ and $G$ are distinct senses of value):

\section{Polarity}

If $x$ is F-good and $y$ is G-bad, then $x$ is F-better than $y$ is G-valuable. If $x$ is F-neutral and $y$ is G-bad, then $x$ is F-better than $y$ is G-valuable. If $x$ is F-good and $y$ is G-neutral, then $x$ is F-better than $y$ is G-valuable.

I use "G-valuable" here rather than "G-good" since it is assumed that $y$ is not G-good but G-bad or G-neutral.

\section{"Very" modification}

If $x$ is very F-good and $y$ is G-good but not very G-good, then $x$ is F-better than $y$ is G-good.

The last constraint can be generalized to other modifiers:

\section{Degree modification}

If $x$ is D F-good and $y$ is $\mathrm{D}^{\prime}$ G-good and D is a higher degree modifier than $\mathrm{D}^{\prime}$, then $x$ is F-better than $y$ is G-good,

where $\mathrm{D}$ is a degree modifier, such as not very, very, extremely, and moderately, and

$\mathrm{D}$ is a higher degree modifier than $\mathrm{D}^{\prime}=\mathrm{df}$. for all $x, y$, and $\mathrm{F}$, if $x$ is $\mathrm{D}$ $\mathrm{F}$ and $y$ is $\mathrm{D}^{\prime}$, then $x$ is F-er than $y$. 
Here are some illustrations:

(21) This table is a better table than Trump is a president, since this table is a good table and Trump is a bad president. (Polarity)

(22) You are more prudent than you are moral, since you are very prudent and you are moral but not very moral. ('Very' modification)

(23) I am more kind than I am beautiful, since I am extremely kind and I am beautiful but not extremely beautiful. (Degree modification)

There is one important complication here that needs to be addressed. When I say that $x$ is F-good but not very F-good and $y$ is very F-bad, I take this to imply that $x$ is F-better than $y$ is F-valuable (by the first polarity principle listed above). But it also implies a cross-polar comparison: $x$ is less F-good than $y$ is F-bad, or, equivalently, $y$ is more F-bad than $x$ is F-good. This comparison holds, since $y$ is very F-bad, while $x$ is F-good but not very F-good. Suppose, for example, that $x$ is a good but not very good table, and $y$ is a very bad philosopher; then $x$ is a better table than $y$ is a philosopher. But it is also true that $x$ is less good as a table than $y$ is bad as a philosopher, or, equivalently, $y$ is worse as a philosopher than $x$ is good as a table, since $x$ is good but not very good as a table, and $y$ is very bad as a philosopher.

Note that cross-polar comparisons can be found in the intra-category cases too. Suppose that your trifling pleasure is good but not very good, and my suffering is very bad. So, your pleasure is better than my suffering, but it is also true that your pleasure is less good than my suffering is bad, or, equivalently, my suffering is worse than your pleasure is good. ${ }^{7}$

\section{Conjunctive Accounts}

Let us now consider the so-called conjunctive accounts of cross-cats. The most prominent and elaborated one is Ewan Klein's (1980). This account, adjusted to my terminology, holds that

" $x$ is F-er than $y$ is G" is true iff there is a degree modifier D such that " $x$ is $\mathrm{D} \mathrm{F}$, and $y$ is not $\mathrm{D} \mathrm{G}$ " is true (where " $\mathrm{D}$ " is read as "at least $\mathrm{D}$ ")

Applied to our initial evaluative sentences, (1)-(3), the results would be as follows:

(24) "This table is a better table than Trump is a president" is true iff there is a degree modifier D such that "this table is a D good table and Trump is not a D good president" is true.

(25) "You are more prudent than you are moral" is true iff there is a degree modifier D such that "you are D prudent and you are not D moral" is true. 
(26) "I am more kind than I am beautiful" is true iff there is a degree modifier D such that "I am D kind and I am not D beautiful" is true.

As seen, Klein's account takes Degree modification and turns it into a definition of cross-cats. I think this account is on the right track - after all, it gives the right verdict about (1) to (3) - but there are still some issues. First, the account seems to overgenerate cross-cats. Suppose that I am very prudent and my sock, unsurprisingly, is not moral and thus not very moral either. Then the account would imply that I am more prudent than my sock is moral. But this is false since my sock cannot be moral. An easy fix would be to say that " $x$ is D F and $y$ is not D G" in the analysans and should be replaced with " $x$ is D F and $y$ is G but not D G."

But that would lead to other problems, for now the account can no longer say that this table is a better table than Trump is a president. Since Trump is a bad president, he does not fulfill the condition "is good but not D good," which he has to fulfill according to the revised account.

Second, Klein's account (including the revised version) has problems satisfying Polarity. Suppose, for instance, that this table is neither good nor bad as a table but is simply neutral as a table. We still seem to be able to say truthfully that this table is better as a table than Trump is good as a president. But there is no D such that this table is $\mathrm{D}$ good, for it is neutral.

Third, it is not clear how to define cross-categorical equality in Klein's account.

\section{My Account}

My account builds on Klein's but avoids its limitation. To see what my account amounts to, let us first introduce the notions of a polarity function, degree modifier function, and modified polarities. A polarity function takes as an input a value kind and spits out a polarity property based on that kind. There are three such functions, pos, neg, and neut. The output of pos is a positive polarity property, a goodness property based on that input value kind, so pos $(\mathrm{F}$-value $)=$ F-goodness. The output of neg is a negative polarity property, a badness property, so neg $(\mathrm{F}$-value $)=$ F-badness. The output of neut is a neutral polarity property, so neut(F-value $)=$ F-neutrality. So, for instance, $\operatorname{pos}($ final value $)=$ being finally good, $n e g($ final value $)=$ being finally bad, and $n e u t$ (final value) $=$ being finally neutral. I am not assuming that all three functions are defined for all kinds of values. Perhaps some kinds of values do not have neutral values (are there neutral hammers?), and perhaps other kinds do not have negative values (e.g., 'moral worth').

A degree modifier function is a function that takes a polarity property as input and spits out a degree-modified polarity property. Examples of degree modifiers functions are very, moderately, barely, somewhat, and not very. So, very $(\mathrm{F}-$ good $)=$ being very F-good, moderately $(\mathrm{F}-\mathrm{bad})$ 


\section{Krister Bykvist}

= being moderately F-bad, barely $(\mathrm{F}-$ good $)=$ being barely F-good, and not very $(\mathrm{F}$-good $)=$ being F-good but not very F-good. I shall assume that there is also a trivial degree modifier function, $t d$, such that $t d(\mathrm{~F}-$ good $)=$ being F-good, $t d(\mathrm{~F}-\mathrm{bad})=$ being F-bad, and $t d(\mathrm{~F}$-neutral $)=$ being F-neutral. $t d$ is the only degree modifier function that is defined for neutral value properties since neutrality does not come in degrees. ${ }^{8}$ Finally, $\mathrm{d}$ is a higher degree function than $\mathrm{d}^{\prime}$ iff, for all $\mathrm{x}, \mathrm{y}$, and F-value, if $\mathrm{x}$ falls under $\mathrm{d}(\mathrm{F})$ and $\mathrm{y}$ falls under $\mathrm{d}^{\prime}(\mathrm{F})$, then $\mathrm{x}$ is F-better than $\mathrm{y}$.

A degree-modified polarity property can be seen as a range property since it carves out a set of items that could differ in degrees of the relevant value but share the property of being within the range. So, the items that are very F-good can differ in F-value, but they all share the property of falling within the range defined by being very F-good. For example, among the very good presidents, some are better presidents than others. A degree-modified polarity property is also non-positional, since whether an item is top, bottom, or $n$th in the relevant ranking is not determined by whether it exemplifies under it. That a flute is very good does not say anything about whether it is best, worst, or $n$th in the ranking of flutes.

It is important to note that the way a value adjective, such as "very F-good," picks out a range property can be a highly contextual matter. What falls under "very F-good" in one context need not fall under "very F-good" in another context. I can count as a very good basketball player in a context in which the comparison class is my elderly relatives, but not in a context in which the comparison class is the Swedish national basketball team. In my statements of the polarity and degree modifier functions, I have ignored these contextual issues to avoid unnecessarily cluttering the exposition.

Here are some examples of degree-modified polarity properties:

Degree-modified positive polarity properties: being kind, being very kind, being finally good, being moderately finally good, being barely prudentially good, being good as a president, being extremely good as a president

Trivially degree-modified neutral polarity properties: being finally neutral, being prudentially neutral

Degree-modified negative polarity properties: being unkind, being very unkind, being selfish, being moderately selfish, being finally bad, being extremely finally bad, being prudentially bad, being somewhat prudentially bad, being very bad as a president, being extremely bad as a president

Finally, a modified polarity function, $m p$, is a function composed of a polarity function, $p$, and a degree function, $d: m p(\cdot)=d(p(\cdot))$. Examples are very $(\operatorname{pos}(\cdot))$, moderately $(n e g(\cdot))$, and $\operatorname{barely}(\operatorname{pos}(\cdot)$. 
We now define cross-categorical betterness in the following way:

$x$ is F-better than $y$ is $\mathrm{G}$-valuable $=\mathrm{df}$. there are modified polarity functions $m p, m p^{\prime}$ such that $x$ falls under $m p^{\prime}$ (F-value), and $y$ falls under $m p^{\prime}(\mathrm{G}-\mathrm{value})$, and $m p$ is of a higher rank than $m p^{\prime}$.

$m p$ is of a higher rank than $m p^{\prime}=\mathrm{df}$. for all $x, y, \mathrm{~F}$, if $x$ falls under $m p(\mathrm{~F}-$ value) and $y$ falls under $m p^{\prime}$ (F-value), then $x$ is F-better than $y$.

Note that this definition ensures that higher rank is irreflexive and asymmetric, given a certain instantiation condition: for any pairs of $m p$ and $m p$ ', there are $\mathrm{F}, x$, and $y$ such that $x$ falls under $m p$ (F-value), and $y$ falls under $m p^{\prime}$ (F-value). We can then use the irreflexivity and asymmetry of F-betterness to show that higher rank must also be irreflexive and asymmetric. To show irreflexivity, suppose for reductio that $m p$ is of a higher rank than itself. Then, given the instantiation condition, there are $\mathrm{F}, x$ such that $x$ falls under $m p$ (F-value), and, given the definition of higher rank, $x$ is F-better than itself, which is impossible. To show asymmetry, suppose for reductio that $m p$ is of a higher rank than $m p^{\prime}$ and $m p^{\prime}$ is of a higher rank than $m p$. Then, given the instantiation condition, there are F, $x, y$ such that $x$ falls under $m p$ (F-value), and $y$ falls under $m p^{\prime}$ (F-value), and, given the definition of higher rank, $x$ is F-better than $y$ and $y$ is F-better than $x$, which is impossible.

If we also assume that for any n-tuple of modified polarities, $m p_{1}$, $m p_{2}, m p_{3}, \ldots, m p_{\mathrm{n}}$, there are $\mathrm{F}, x_{1}, x_{2}, x_{3}, \ldots, x_{\mathrm{n}}$ such that $x_{1}$ falls under $m p_{1}($ F-value $), x_{2}$ falls under $m p_{2}($ F-value $), x_{3}$ falls under $m p_{3}(\mathrm{~F}-$ value),..., and $x_{n}$ falls under $m p_{n}$ (F-value), we can also show that higher rank must be acyclical, if we also assume that F-betterness itself must be acyclical, which seems plausible for any F. ${ }^{9}$ Suppose for reductio that $m p_{1}$ is of a higher rank than $m p_{2}, m p_{2}$ is of a higher rank than $m p_{3}, \ldots$, but $m p_{\mathrm{n}}$ is of a higher rank than $m p_{1}$. Suppose also that $x_{1}$ falls under $m p_{1}(\mathrm{~F}), x_{2}$ falls under $m p_{2}(\mathrm{~F}), x_{3}$ falls under $m p_{3}(\mathrm{~F}), \ldots$, and $x_{\mathrm{n}}$ falls under $m p_{\mathrm{n}}$. Then, by the definition of higher rank, $x_{1}$ is F-better $x_{2}$, $x_{2}$ is F-better than $x_{3}, x_{3}$ is F-better than $x_{4}, \ldots$, but $x_{\mathrm{n}}$ is F-better than $x_{1}$, which is impossible.

Admittedly, these instantiation conditions assume a certain richness in value structure that could be questioned. An alternative way to ensure that higher rank is well-behaved is simply to postulate that the relation is irreflexive, asymmetric, and transitive.

Note also that Cross-Cat Betterness ensures the following:

\section{Value Category Harmony}

For all $\mathrm{F}, x$, and $y$, if there are $m p$ and $m p^{\prime}$ such that $x$ falls under $m p$ (F-value), and $y$ falls under $m p^{\prime}$ (F-value), and $m p$ is of a higher rank than $m p^{\prime}$, then there are no $m p^{\prime \prime}$ and $m p^{\prime \prime}$ such that $x$ falls under $m p^{\prime \prime}(\mathrm{F})$, and $y$ under $m p^{\prime \prime \prime}(\mathrm{F})$, and $m p^{\prime \prime}$ is of a higher rank than $m p "$. 


\section{Krister Bykvist}

For if this conditional is false, it follows from Cross-Cat Betterness that for some F, $x$, and $y, x$ is F-better than $y$ and $y$ is F-better than $x$, which is impossible since F-betterness is asymmetric.

What about cross-categorical equality? We cannot just say that

$x$ is as F-valuable as $y$ is G-valuable iff there is an $m p$ such that $x$ falls under $m p$ (F-value) and $y$ falls under $m p$ (G-value).

This account of equality would entail that

$x$ is as kind as $y$ is beautiful, whenever $x$ is kind and $y$ is beautiful, no matter whether $x$ is kind but not very kind and $y$ is very beautiful.

$x$ is as good a table as $y$ is a president, whenever the $x$ is a good table and $y$ is a good president, no matter whether $x$ is not a very good table and $y$ is a very good president.

This captures a very coarse-grained notion of cross-cat equality. At the other extreme, we have exact cross-cat equality:

$x$ is as F-valuable as $y$ is G-valuable iff there are $m p$ and $m p$ ' such that $x$ falls under $m p$ (F-value), and $y$ falls under $m p^{\prime}$ (G-value), and $m p$ has the same rank as $m p$ '.

$m p$ has the same rank as $m p^{\prime}=\mathrm{df}$. for all $x, y$, and $\mathrm{F}$, if $x$ falls under $m p$ (F-value) and $y$ falls under $m p^{\prime}(\mathrm{F}$-value), then $x$ is exactly as F-valuable as $y$.

This is a very restrictive notion of cross-cat equality, however. Indeed, it seems as though $m p$ has the same rank as $m p^{\prime}$ only if $m p=m p^{\prime}=$ $d t($ neut $(\cdot))$, since any other modalized polarity function will allow for variation in the degree of F-value. If this is correct, $x$ is as F-valuable as $y$ is G-valuable only if $x$ is F-neutral and $y$ is G-neutral.

Here is a better account that avoids this problem:

Cross-Cat Equality

$\mathrm{x}$ is as F-valuable as $y$ is G-valuable iff, for all $m p, x$ falls under $m p(\mathrm{~F}-$ value) iff $y$ falls under $m p$ (G-value) (and both $x$ and $y$ fall under some $m p)$.

This definition makes cross-categorical equality transitive, which is an attractive feature. Note also that it entails that Mozart is as good a composer as Michelangelo is a sculptor (they are both exceptionally good in their own fields), which seems to be a welcome result. Indeed, since this holds even though we cannot say that Mozart is a more creative artist than Michelangelo, or that Michelangelo is a more creative artist than Mozart, or that they are equally creative, one may suggest that this captures a notion of parity. (I say $a$ notion rather than the notion since there seem to be many different value concepts one can have in mind when one talks about parity. $)^{10}$ 
Let us now turn to inter-polar cross-cats. I suggest the following definitions:

$x$ is more F-good than $y$ is G-bad $=\mathrm{df}$. there are modified polarity functions $m p, m p^{\prime}$, such that $m p=d(p o s(\cdot))$ and $m p^{\prime}=d^{\prime}(n e g(\cdot)), d$ is a higher degree modifier function than $d^{\prime}$, and $x$ falls under $m p$ (F-value), and $y$ falls under $m p^{\prime}$ (G-value).

$x$ is more F-bad than $y$ is G-good $=\mathrm{df}$. there are modified polarity functions $m p, m p^{\prime}$, such that $m p=d(n e g(\cdot))$ and $m p^{\prime}=d^{\prime}(p o s(\cdot)), d$ is a higher degree modifier function than $d^{\prime}$, and $x$ falls under $m p$ (F-value) and $y$ falls under $m p^{\prime}$ (G-value).

$x$ is as F-bad as $y$ is G-good $=\mathrm{df}$. for all $m p$ and $m p^{\prime}$ such that $m p=$ $d(n e g(\cdot))$ and $m p^{\prime}=d^{\prime}(\operatorname{pos}(\cdot)), d=d^{\prime}, x$ falls under $m p($ F-value $)$ iff $y$ falls under $m p^{\prime}(\mathrm{G}-\mathrm{v}$ alue).

Since my aim in this chapter is modest - just to outline an account of evaluative cross-cats - I will not say much about how to generalize my account to all non-evaluative cross-cats. Suffice it to say that the central notions, i.e., polarity functions and degree modifier functions, which together define modified polarity functions, can easily be generalized to non-evaluative properties. I have already done so for degree modifier functions, but polarity is also a general aspect of gradable adjectives (and the expressed properties). For example, length has both a positive polarity, being tall, and a negative one, being short. Similarly, weight has a positive polarity, being heavy, as well as a negative one, being light. Felt temperature seems to have three polarities: warm, cold, and neutral. (Of course, in all these cases, polarity is a contextual matter.) The polarity functions pol, neg, neut can thus be extended to have non-evaluative kinds as inputs, and the previous definitions can be extended to apply to non-evaluative cross-cats, mutatis mutandis.

The advantages of my account are the following:

(1) It has the right entailment.

If $x$ is F-better than $y$ is G-good and $y$ is very G-good, then $x$ is at least very F-good.

(2) It applies even when there are gaps in one ordering.

If $x$ falls under $m p$ (F-value) and $y$ falls under $m p^{\prime}$ (G-value), and $m p$ is of a higher rank than $m p^{\prime}$, then $x$ is F-better than $y$ is G-valuable, even if either the F-value ranking or the G-value ranking is gappy.

(3) It applies even when the number of F-value ranked items is not the same as the number of G-value ranked items.

If $x$ falls under $m p$ (F-value), and $y$ falls under $m p^{\prime}(\mathrm{G}$-value), and $m p$ is of a higher rank than $m p^{\prime}$, then $x$ is F-better than $y$ is $\mathrm{G}$-valuable, even if the number of items that have F-value is different from the number of items that have G-value. 
(4) It applies even when the F-value ranking or G-value ranking is unbounded.

If $x$ falls under $m p$ (F-value) and $y$ falls under $m p^{\prime}(\mathrm{G})$ and $m p$ is of a higher rank than $m p^{\prime}$, then $x$ is F-better than $y$ is G-valuable, even if there is no maximum or minimum in one or both of the rankings.

(5) It satisfies Polarity.

This is obvious.

(6) It satisfies Degree modification.

This is also obvious.

(7) It deals with inter-polar cross-cats.

This is also obvious.

\section{Normative Applications}

Now that we have a better grasp of evaluative cross-cats, it is time to see whether they have any normative relevance. Even though these new value relations would of course be usable in purely axiological matters when we are just interested in making value judgments, it would be disappointing if these value relations could not be put to any normative use at all.

One possible application is normative decisions based on comparisons of what is good for different people. ${ }^{11}$ Suppose you are convinced, as many economists still seem to be, that there is no common value dimension on which we can compare what is good for people, as well-being is interpersonally incomparable. Then saying that $x$ is better for me than $y$ is for you amounts to a cross-cat. You can then use my analysis to decide what you ought to do in a choice between realizing A, which is very good for me, and realizing $\mathrm{B}$, which is good but not very good for you (and no other person is affected). Since, according to my analysis, A is better for me than B is for you, this can be seen as providing a reason to choose A over B.

A second application is this. We have two positions and one applicant. One position requires prudence and the other being moral. We need to offer the candidate one of the positions. If the candidate is more prudent than moral - perhaps because she is very prudent but not very moral then we should offer her the job that requires prudence. Now some might object that we are invoking another value consideration here: finding the best match between the candidate and the job. But this does not take away the significance of the cross-categorical value comparison since we are not using the "best match" value to define cross-categorical value comparisons; rather, the "best match" value is based on the cross-categorical comparison.

Another application relies on the link between values and attitudes. Even though it is controversial to define values in terms of attitudes, it is reasonable to assume that, at least for some kinds of values, if something 
has a certain value, it is fitting to have a certain attitude toward it. What attitude it is fitting to have toward it depends on what kind of value it has. Sometimes it is obvious what attitude it would be fitting to have: for example, it is fitting to admire what is admirable, envy what is enviable, praise what is praiseworthy, blame what is blameworthy, and desire what is desirable.

Cross-cats can be put to use here. If $x$ is more admirable than $y$ is enviable - perhaps because $x$ is very admirable and $y$ is enviable but not very enviable, then to admire $x$ but not envy $y$ seems a more fitting combined stance than not to admire $x$ but envy $y$. Furthermore, the stance "admire $x$ very much, envy $y$ but not very much" is more fitting than the stance "admire $x$ but not very much, envy $y$ very much." More generally, if $x$ is more admirable than $y$ is enviable, it is more fitting to admire $x$ more than one envies $y$ than to envy $y$ more than one admires $x$. Note that this claim invokes yet another kind of cross-categorical comparison - namely, one of attitudinal strength since the strength of admiration and the strength of envy do not seem to be reducible to one common kind of attitudinal strength. ${ }^{12}$

The final application is evaluative uncertainty. The obvious obstacle to taking evaluative uncertainty seriously is that we can be uncertain about the truth of two radically different theories that employ different kinds of values. For example, we can be undecided between (a) a certain virtue theory (e.g., Philippa Foot's) according to which there is no impartial value - we can only talk about virtuous or vicious acts, and (b) a utilitarian theory according to which actions can be (instrumentally) impartially good, but no actions are virtuous or vicious. Suppose that the choice situation is like the following (without well-defined probabilities):

\begin{tabular}{lll}
\hline Actions & Virtue Theory & Utilitarianism \\
\hline A & Very vicious & Very impartially good \\
B & Very virtuous & $\begin{array}{l}\text { Impartially good but not very } \\
\text { impartially good }\end{array}$ \\
\hline
\end{tabular}

Action A might be the joyful killing of an innocent that brings about billions of extra very happy lives. Action B is the joyful omission of killing an innocent that brings about a few extra very happy lives created by the agent out of the most virtuous motives. There are no direct value comparisons to be made across the theories since the theories employ different value concepts, but we still seem to be able to say the following:

A is impartially better, according to utilitarianism, than it is virtuous, according to virtue theory, since it is impartially very impartially good according to utilitarianism and very vicious according to virtue theory.

$\mathrm{B}$ is more virtuous, according to virtue theory, than it is impartially good, according to utilitarianism, since it is very virtuous according 


\section{Krister Bykvist}

to virtue theory and impartially good but not very impartially good according to utilitarianism.

$\mathrm{B}$ is impartially better, according to utilitarianism, than A is virtuous, according to virtue theory, since B is impartially good but not very impartially good according to utilitarianism and A is very vicious according to virtue theory.

These cross-cats seem to suggest that B would be a more reasonable choice since it "maximizes the minimum cross-categorical value."

More generally, the idea is that it is reasonable to choose B in a situation of the following kind (without well-defined probabilities).

\begin{tabular}{lll}
\hline Actions & Theory $T 1$ & Theory T2 \\
\hline A & Very T1-bad & Very T2-good \\
B & Very T1-good & T2-good but not very T2-good \\
\hline
\end{tabular}

Of course, maximin is not uncontroversial, but this is just one example of how cross-cats can be used to guide action in certain cases of evaluative uncertainty. Other principles that take into account both (qualitative) probability or plausibility and ordinal value information can be used instead. The scope of application of this approach to decision-making under evaluative uncertainty goes beyond that of existing approaches, since they tend to assume either that no value comparisons can be made across theories or that they can made only when the theories employ exactly the same value concept. ${ }^{13}$

\section{Concluding Remarks}

The conclusion is that, notwithstanding the old saying, we can compare apples with oranges! Joking aside, there seems to be room for cross-cats that can be put to normative use. Of course, much more needs to be said to bolster this claim and show how it fits into a general account of crosscats, both evaluative and non-evaluative. I have provided only a very rough sketch of how to make sense of evaluative cross-cats. One issue that needs to be addressed is that cross-cats seem more natural and plausible when they involve similar value concepts, such as good as a table and good as a president, or prudentially good and morally good; indeed, one could claim that there is a specific second-order value concept that unifies the value concepts in each pair: goodness of its kind, and goodness of persons, respectively. Cross-cats that invoke very different concepts and very different value bearers seem less natural and plausible. For example, it seems odd to say that my excellent wine is a better wine than my having a dull life is a good state of affairs. So, one option is to add 
restrictions to my account. For $x$ to be cross-categorically comparable to $x$ and $y$, they have to fall under similar (but nonidentical) value concepts and be similar value bearers. Of course, for this to be useful, we need to decide what "similar" means.

Another remaining question is why cross-cats seem more compelling when they involve objects with extremal values. It is very compelling to think that $x$ is F-better than $y$ is G-valuable when $x$ is extremely F-good and the very best $\mathrm{F}$ one can imagine, whereas $y$ is extremely G-bad and the very worst $\mathrm{G}$ one can imagine. ${ }^{14}$ We know this cannot just be a question of their relative positions, so we need some other explanation of the compellingness.

Even though there are remaining issues, I hope that I have at least shown that cross-categorical comparisons should be taken seriously and not simply dismissed as ungrammatical, meaningless, or merely metaphorical - and that my account is an improvement on the existing ones. ${ }^{15}$

\section{Notes}

1 I assume that prudent, moral, kind, and beautiful are thick evaluative terms.

2 It might be clearer to say, "than Trump is good as a president."

3 Kraut (2011: 153) claims that it makes sense to say, "This watermelon is a better watermelon than that poet is a poet," but he does not provide an account of such comparisons.

4 For more on the notion of covering value, see Chang (1997).

5 See Chang (2002).

6 The (in)famous zero-one rule for interpersonal comparisons of well-being, according to which everyone's top-ranked options are assigned 1 and everyone's bottom-ranked options are assigned 0 , satisfies an analogous principle and faces problems analogous to those that afflict the positional account. For more on critical discussion of the zero-one rule, see Hausman (1995).

7 Here "less good" does not mean "worse," which it can do in some contexts.

8 These degree modifier functions are the standing meanings of their linguistic counterparts "very," "moderately," "barely," and so on. A linguistic degree modifier, such as "very," retains its meanings across different constructions, even if it is applied to different evaluative adjectives. For example, "very" has the same meaning in "very prudentially good" as in "very finally good."

9 At least this holds for the notion of betterness that is the comparative of "good," which is my focus here. For an alternative non-transitive notion of betterness, see Temkin (2012: ch. 1).

10 For more on the notion of parity, see Chang (2002).

11 I would like to thank Gustaf Arrhenius and Michael Morreau for suggesting this application.

12 See Rabinowicz (2012: 153) for a brief discussion of what he calls "crosskind" commensuration of attitudes.

13 MacAskill et al. (2020) offers an opinionated introduction to existing accounts of decision-making under moral uncertainty.

14 I would like to thank Joe Roussos and Wlodek Rabinowicz for alerting me to this. 


\section{0}

Krister Bykvist

15 A previous version of this paper was presented at a workshop on nonstandard comparisons of value, at the Institute for Futures Studies, Stockholm, 6 December 2019. I am grateful for comments from the audience. I am especially grateful to the editors of this volume Henrik Andersson and Anders Herlitz, and to Chrisoula Andreou, Gustaf Arrhenius, Erik Carlson, Ruth Chang, Ralf Bader, Zak Kopeikin, Michael Morreau, Wlodek Rabinowicz, and Joe Roussos.

\section{References}

Bale, A. (2006), The Universal Scale and the Semantics of Comparison, PhD thesis (McGill University).

Bale, A. (2008), 'A Universal Scale of Comparison', Linguistics and Philosophy 31: 1 .

Chang, R. (1997), 'Introduction', in R. Chang (ed.), Incommensurability, Incomparability, and Practical Reason (1-34). Harvard University Press.

Chang, R. (2002), 'The Possibility of Parity', Ethics 112/4: 659-688.

Hausman, D. (1995), 'The Impossibility of Interpersonal Utility Comparisons', Mind 104/415: 473-490.

Klein, E. (1980), 'The Semantics of Positive and Comparative Adjectives', Linguistics and Philosophy 4: 1-45.

Kraut, R. (2011), Against Absolute Goodness (Oxford University Press).

MacAskill, W., Bykvist, K., and Ord, T. (2020), Moral Uncertainty (Oxford University Press).

Rabinowicz, W. (2012), 'Value Relations Revisited', Economics and Philosophy 28/2: 133-164.

Temkin, L., (2012), Rethinking the Good (Oxford University Press). 